\title{
Max Entropy through Natural Interactions ${ }^{\dagger}$
}

\author{
Yair Neuman ${ }^{1}$ and Yochai Cohen ${ }^{2}$ \\ 1 Department of Cognitive and Brain Sciences and the Zlotowski Center for Neuroscience, Ben-Gurion \\ University of the Negev, Beer-Sheva, Israel \\ 2 Gilasio Coding, 6458701 Tel-Aviv, Israel \\ † Presented at the Entropy 2021: The Scientific Tool of the 21st Century, 5-7 May 2021; Available online: \\ https://sciforum.net/conference/Entropy2021/.
}

Published: 5 May 2021

The Principe of Maximum Entropy, suggests that when one tries to predict the shape of a distribution, then among all possible distributions available for his choice, he should choose the one that maximizes the entropy of the distribution under some few chosen constraints expressing his limited knowledge of the situation. This principle has deep meaning for human and non-human organisms alike, but it is hard to imagine how it is taken place in natural environments under bounded rationality [1]. The context of the current talk is the way natural cognitive processes may be modeled through entropy and bounded rationality (e.g., [2,3]). More specifically, we would like to present a novel idea [4] describing the way in which the entropy of a predicted distribution increases through a structured process of natural interaction that builds on three principles only: Zipf's [5] principle of least effort, Laplace's principle of indifference, and The Copernican principle, suggesting no observer occupy a special place in the universe. This process will be presented, illustrated and supported by simulations never presented before.

\section{References}

1. Simon, H.A. Models of Man; Wiley: New York, NY, USA, 1957.

2. Neuman, Y.; Vilenchik, D. Modeling small systems through the relative entropy lattice. IEEE Access 2019, 7, 43591-43597.

3. Neuman, Y.; Cohen, Y.; Tamir, B. Short-term prediction through ordinal patterns. R. Soc. Open Sci. 2021, 8, 201011.

4. Neuman, Y. How Small Social Systems Work: From Soccer Teams to Families and Jazz Trios; Springer: New York, NY, USA, 2021.

5. Zipf, G.K. Human Behavior and the Principle of Least Effort: An Introduction to Human Ecology; Ravenio Books: 2016.

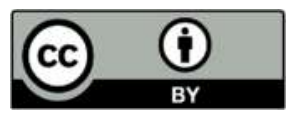

(C) 2021 by the authors. Licensee MDPI, Basel, Switzerland. This article is an open access article distributed under the terms and conditions of the Creative Commons Attribution (CC BY) license (http://creativecommons.org/licenses/by/4.0/). 